\title{
Geometric Spanners With Small Chromatic Number ${ }^{\star}$
}

\author{
Prosenjit Bose ${ }^{1}$, Paz Carmi ${ }^{1}$, Mathieu Couture ${ }^{1}$, Anil Maheshwari ${ }^{1}$, Michiel Smid ${ }^{1}$, and Norbert Zeh ${ }^{2}$ \\ 1 School of Computer Science, Carleton University \\ 2 Faculty of Computer Science, Dalhousie University
}

\begin{abstract}
Given an integer $k \geq 2$, we consider the problem of computing the smallest real number $t(k)$ such that for each set $P$ of points in the plane, there exists a $t(k)$-spanner for $P$ that has chromatic number at most $k$. We prove that $t(2)=3, t(3)=2, t(4)=\sqrt{2}$, and give upper and lower bounds on $t(k)$ for $k>4$. We also show that for any $\epsilon>0$, there exists a $(1+\epsilon) t(k)$-spanner for $P$ that has $O(|P|)$ edges and chromatic number at most $k$. Finally, we consider an on-line variant of the problem where the points of $P$ are given one after another, and the color of a point must be assigned at the moment the point is given. In this setting, we prove that $t(2)=3, t(3)=1+\sqrt{3}, t(4)=1+\sqrt{2}$, and give upper and lower bounds on $t(k)$ for $k>4$.
\end{abstract}

\section{Introduction}

Let $P$ be a set of $n$ points in the plane. A geometric graph with vertex set $P$ is an undirected graph whose edges are line segments that are weighted by their Euclidean length. For a real number $t \geq 1$, such a graph $G$ is called a $t$-spanner if the weight of the shortest path in $G$ between any two vertices $p$ and $q$ does not exceed $t|p q|$, where $|p q|$ is the Euclidean distance between $p$ and $q$. The smallest $t$ having this property is called the stretch factor of the graph $G$. Thus, a graph with stretch factor $t$ approximates the $\left(\begin{array}{l}n \\ 2\end{array}\right)$ distances between the points in $P$ within a factor of $t$. The problem of constructing $t$-spanners with $O(n)$ edges for any given point set has been studied intensively; see the book by Narasimhan and Smid [5] for an overview.

In this paper, we consider the problem of computing $t$-spanners whose chromatic number is at most $k$, for some given value of $k$. The goal is to minimize the value of $t$ over all finite sets $P$ of points in the plane. We call a spanner whose chromatic number is at most $k$ a $k$-chromatic spanner.

Problem 1. Given an integer $k \geq 2$, let $t(k)$ be the infimum of all real numbers $t$ with the property that for every finite set $P$ of points in the plane, a $k$-chromatic $t$-spanner for $P$ exists. Determine the value of $t(k)$.

Observe that in the definition of $t(k)$, there is no requirement on the number of edges of the chromatic spanner. This is not a restriction, because, as shown by Gudmundsson et al. [4], any $t$-spanner for $P$ contains a subgraph with $O(n)$ edges which is a $((1+\epsilon) t)$-spanner for $P$.

We show how to obtain a 2 -chromatic 3 -spanner for any point set $P$, thus showing that $t(2) \leq 3$. We also give an example of a point set $P$ such that any 2-chromatic graph with vertex set $P$ has stretch factor at least three. Thus, we have $t(2)=3$.

Next, we show how to compute a 3 -chromatic 2 -spanner of any point set $P$, thereby proving that $t(3) \leq 2$. We also show, by means of an example, that $t(3) \geq 2$. Thus, we obtain that $t(3)=2$. For $k=4$, we show how to compute a 4 -chromatic $\sqrt{2}$-spanner of any point set $P$; thus $t(4) \leq \sqrt{2}$. Again by means of an example, we also show that $t(4) \geq \sqrt{2}$. Therefore, we have $t(4)=\sqrt{2}$.

For $k>4$, we are not able to obtain the exact value of $t(k)$. Inspired by the ordered $\Theta$-graph of Bose et al. [1], we show that $t(k) \leq 1+2 \sin \frac{\pi}{2(k-1)}$. We also show that the vertex set of the regular $(k+1)$-gon gives $t(k) \geq 1 / \cos \frac{\pi}{k+1}$.

In the second part of the paper, we consider an on-line variant of the problem where the points of $P$ are given one after another, and the color of a point must be assigned at the moment when the point is given; thus, later on, the color of a point cannot be changed. This makes the problem more difficult. Consequently, the bounds are higher, but still tight for $k=2,3,4$. All our bounds are summarized in Table 1.

\footnotetext{
* Research partially supported by HPCVL, NSERC, MRI, CFI, and MITACS.
} 
Problem 2. Given an integer $k \geq 2$, let $t^{\prime}(k)$ be the infimum over all real numbers $t$ with the following property: For every finite set $P$ of points in the plane that is given on-line, assign a color from $\{1,2, \ldots, k\}$ to each point at the moment it is given, such that the complete $k$-partite graph with vertex set $P$ induced by this coloring is a $t$-spanner of $P$. Determine the value of $t^{\prime}(k)$.

A simple variant of the ordered $\Theta$-graph shows that $t^{\prime}(k) \leq 1+2 \sin (\pi / k)$. Thus, we have $t^{\prime}(2) \leq 3$, $t^{\prime}(3) \leq 1+\sqrt{3}$ and $t^{\prime}(4) \leq 1+\sqrt{2}$. Since $t^{\prime}(2) \geq t(2)=3$, it follows that $t^{\prime}(2)=3$. We also give examples showing that $t^{\prime}(3) \geq 1+\sqrt{3}$ and $t^{\prime}(4) \geq 1+\sqrt{2}$. We finally show that, for $k \geq 5, t^{\prime}(k) \geq 1 / \cos \frac{\pi}{k}$.

The rest of this paper is organized as follows: in Section 2, we define the $t$-ellipse property and show its relationship to our problem. In Section 3, we give upper and lower bounds for the off-line problem (Problem 1). In Section 4, we give upper and lower bounds for the on-line problem (Problem 2). In Section 5, we present simulation results. We conclude in Section 6. In Table 1, we summarize our results. We now motivate our work.

\begin{tabular}{|c|c|c|c|c|}
\hline number of colors & \multicolumn{2}{|c|}{$t(k)$ (off-line) } & \multicolumn{2}{c|}{$t^{\prime}(k)$ (on-line) } \\
\hline$k$ & lower bound & upper bound & lower bound & upper bound \\
\hline 2 & 3 & 3 & 3 & 3 \\
\hline 3 & 2 & 2 & $1+\sqrt{3}$ & $1+\sqrt{3}$ \\
\hline 4 & $\sqrt{2}$ & $\sqrt{2}$ & $1+\sqrt{2}$ & $1+\sqrt{2}$ \\
\hline$k$ & $1 / \cos \frac{\pi}{k+1}$ & $1+2 \sin \frac{\pi}{2(k-1)}$ & $1 / \cos \frac{\pi}{k}$ & $1+2 \sin \frac{\pi}{k}$ \\
\hline
\end{tabular}

Table 1. Summary of our results.

Motivation: In a recent paper, Raman and Chebrolu [6] proposed a new protocol, called 2P, allowing to address rural Internet connectivity in a low-cost manner using off-the-shelf 802.11 hardware. Since their infrastructure uses several directional antennae at one node rather than one single omnidirectional antenna, simultaneous communications are possible at one node. However, due to restrictions inherent in the 802.11 standard, backbone nodes have to communicate with each other using a single channel. While simultaneous transmissions and simultaneous receptions are possible, it is not physically possible for one node to both transmit and receive at the same time. Therefore, backbone nodes have to alternate between the send and receive states (see Figure 1). This forces the backbone to be a bipartite graph, i.e., to have chromatic number two.

The backbone creation algorithm of Raman and Chebrolu [6] outputs a tree, which is obviously bipartite. However, the tree structure presents the following disadvantage: it is possible that the path that a message has to follow is much longer than the distance (either Euclidean or in terms of hops) between the originating node and its destination. For example, in Figure 1, a message routed from node 1 to node 3 has to go through nodes 2 and 4, whereas a direct link between 1 and 3 could be added while still satisfying the bipartition requirement.

Note that the physical constraint preventing nodes to simultaneously receive and transmit can be met even if the graph is not bipartite. In fact, any graph with chromatic number $k$ would meet this requirement: all one has to do is to prevent two nodes that have different colors to transmit simultaneously. A degenerate case is when each node has its own color, in which case at most one node can transmit at any given moment. This case is undesirable, since the amount of time during which a node can transmit decreases as the size of the network increases.

For these reasons, it is desirable to have geometric graphs that have both small chromatic number and small stretch factor. 

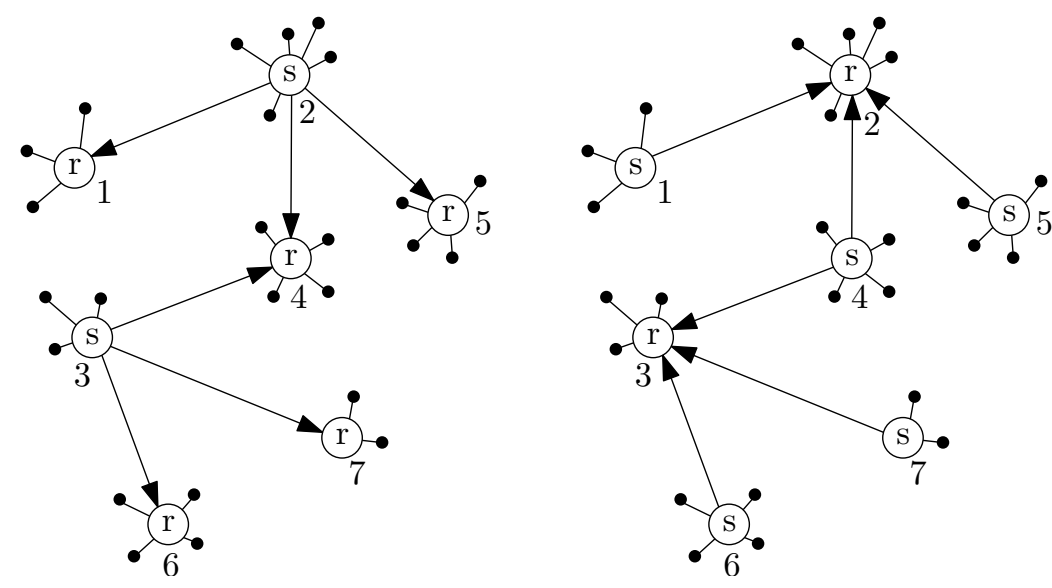

s: send

r: receive

- client nodes

backbone nodes

Fig. 1. The two possible states of the backbone nodes.

\section{The $t$-Ellipse Property}

In this section, we show that Problem 1, i.e., determining the smallest value of $t$ such that a $k$-chromatic $t$-spanner exists for any point set $P$, is equivalent to minimizing the value of $t$ such that any point set can be colored using $k$ colors in a way that satisfies the so-called $t$-ellipse property.

Definition 1 ( $t$-ellipse property). Let $k \geq 2$ be an integer, let $P$ be a finite set of points in the plane and let $c: P \rightarrow\{1, \ldots, k\}$ be a $k$-coloring of $P$. We say that the coloring $c$ satisfies the $t$-ellipse property if, for each pair of distinct points $p$ and $q$ in $P$ with $c(p)=c(q)$, there exists a point $r \in P$ such that $c(r) \neq c(p)$ and $|p r|+|r q| \leq t|p q|$.

Thus, if $p$ and $q$ have the same color, then the ellipse $\left\{x \in \mathbb{R}^{2}:|p x|+|x q| \leq t|p q|\right\}$ contains a point $r$ of $P$ whose color is different from that of $p$ and $q$.

Proposition 1. Let $k \geq 2$, let $P$ be a set of points in the plane, and let $G$ be a $k$-chromatic $t$-spanner of $P$ with $k$-coloring $c$. Then $c$ satisfies the t-ellipse property.

Proof. Let $p, q \in P$ be two points with $c(p)=c(q)$. Since $G$ is a $t$-spanner, there exists a $t$-spanning path $\Pi$ in $G$ from $p$ to $q$. Let $r$ be the point on $\Pi$ that is adjacent to $p$. Since the length of $\Pi$ is at most $t|p q|$, we note that $|p r|+|r q|$ is at most $t|p q|$. Since the edge $(p, r)$ is in $G$, it follows that $c(p) \neq c(r)$. Therefore, $c$ satisfies the $t$-ellipse property.

Proposition 2. Let $k \geq 2$, let $P$ be a set of points in the plane, and let $c: P \rightarrow\{1, \ldots, k\}$ be a $k$-coloring of $P$ that satisfies the $t$-ellipse property. Then, there exists a $k$-chromatic $t$-spanner of $P$.

Proof. Let $K_{c}(P)$ be the complete $k$-partite graph with vertex set $P$ in which there is an edge between two points $p$ and $q$ if and only if $c(p) \neq c(q)$. By definition, $K_{c}(P)$ is $k$-colorable. We show that $K_{c}(P)$ is a $t$-spanner of $P$. Let $p$ and $q$ be two distinct points of $P$ such that $(p, q)$ is not an edge in $K_{c}(P)$. This means that $c(p)=c(q)$. Since $c$ has the $t$-ellipse property, there exists a point $r$ in $P$ such that $c(r) \neq c(p)$ and $|p r|+|r q| \leq t|p q|$. Since $c(r) \neq c(p)$ (and consequently, $c(r) \neq c(q)$ ), the edges $(p, r)$ and $(r, q)$ are both in $K_{c}(P)$. This means that $(p, r, q)$ is a $t$-spanner path in $K_{c}(P)$ between $p$ and $q$.

From this point on, unless specified otherwise, we define the stretch factor of a $k$-coloring of a point set to be the stretch factor of the complete $k$-partite graph induced by this coloring. By Propositions 1 and 2, the problem of determining $t(k)$ is equivalent to determining the minimum stretch factor of any $k$-coloring of any point set. 
We conclude this section by showing why it is sufficient to focus on the coloring problem without worrying about the number of edges in the spanner. The following theorem is due to Gudmundsson et al. [4]; its proof is based on the well-separated pair decomposition of Callahan and Kosaraju [2].

Theorem 1. [4] Let $\epsilon>0$ and $t \geq 1$ be constants, let $P$ be a set of $n$ points in the plane, and let $G$ be a $t$-spanner of $P$. There exists a subgraph $G^{\prime}$ of $G$, such that $G^{\prime}$ is a $((1+\epsilon) t)$-spanner of $P$ and $G^{\prime}$ has $O(n)$ edges.

Proposition 3. Let $k \geq 2$, let $P$ be a set of $n$ points in the plane, and let $c: P \rightarrow\{1, \ldots, k\}$ be a $k$ coloring of $P$ that satisfies the $t$-ellipse property. Then, for any constant $\epsilon>0$, there exists a $k$-chromatic $((1+\epsilon) t)$-spanner of $P$ that has $O(n)$ edges.

Proof. By Proposition 2, there exists a $k$-chromatic $t$-spanner $G$ of $P$. By Theorem $1, G$ contains a subgraph $G^{\prime}$ with $O(n)$ edges, such that $G^{\prime}$ is a $((1+\epsilon) t)$-spanner of $P$. Since $G$ is $k$-chromatic, $G^{\prime}$ is $k$-chromatic as well.

\section{Upper and lower bounds on $t(k)$}

The structure of this section is as follows: For $k=2,3$, and 4, we give coloring algorithms whose outputs have bounded stretch factor. Then, we show that these stretch factors are tight by providing point sets for which no coloring algorithm can achieve a better stretch factor. Then we present our upper and lower bounds for $t(k)$, when $k>4$.

We now give the coloring algorithm for $k=2$.

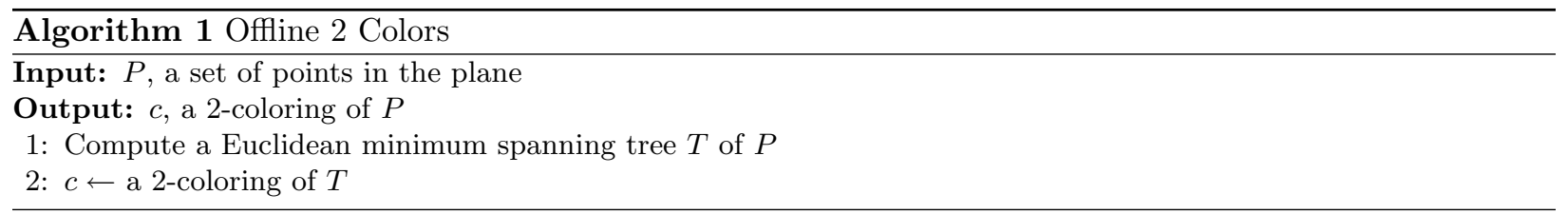

Lemma 1. For any point set P, the 2-coloring computed by Algorithm 1 has stretch factor at most 3. Thus, we have $t(2) \leq 3$.

Proof. It is sufficient to show that the 2-coloring $c$ computed by Algorithm 1 has the 3-ellipse property. Let $p$ and $q$ be two distinct points in $P$ such that $c(p)=c(q)$. Observe that $(p, q)$ is not an edge in the minimum spanning tree $T$. Let $r$ be the nearest neighbor of $p$. Since the edge $(p, r)$ is in $T$, we have $r \neq q$ and $c(r) \neq c(p)$. Since $r$ is closer to $p$ than $q$, we have

$$
|p r|+|r q| \leq|p r|+|r p|+|p q|=2|p r|+|p q| \leq 2|p q|+|p q|=3|p q| .
$$

Lemma 2. For every $\epsilon>0$, there exists a point set $P$ such that every 2 -coloring of $P$ has stretch factor at least $3-\epsilon$. Thus, we have $t(2) \geq 3$.

Proof. Refer to Figure 2 for what follows. Let $n$ be an odd integer, and let $P=\left\{p_{1}, \ldots, p_{n}\right\}$ be the set of vertices of a regular $n$-gon given in counter-clockwise order. Let $c$ be an arbitrary 2-coloring of $P$. By the pigeonhole principle, there are two points in $P$ which are adjacent on the $n$-gon and that have the same color. We may assume without loss of generality that these two points are $p_{1}$ and $p_{2}$. Also, we may assume that $\left|p_{1} p_{2}\right|=1$. Let $t$ be any real number such that $c$ satisfies the $t$-ellipse property. Then $\left|p_{1} p_{3}\right|+1 \leq t$. But $\left|p_{1} p_{3}\right|=2 \sin ((n-2) \pi / 2 n)$, which tends to 2 as $n$ goes to infinity. 


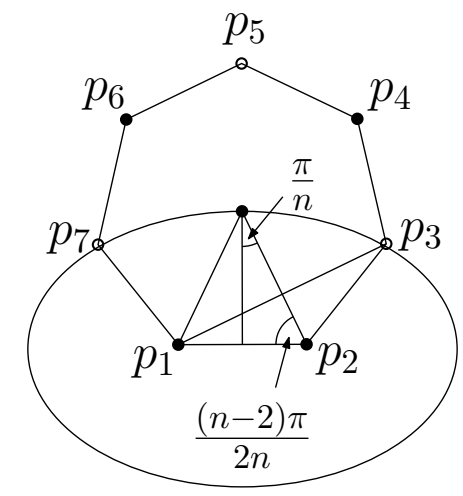

Fig. 2. Lower bound of $3-\epsilon$ for $k=2$.

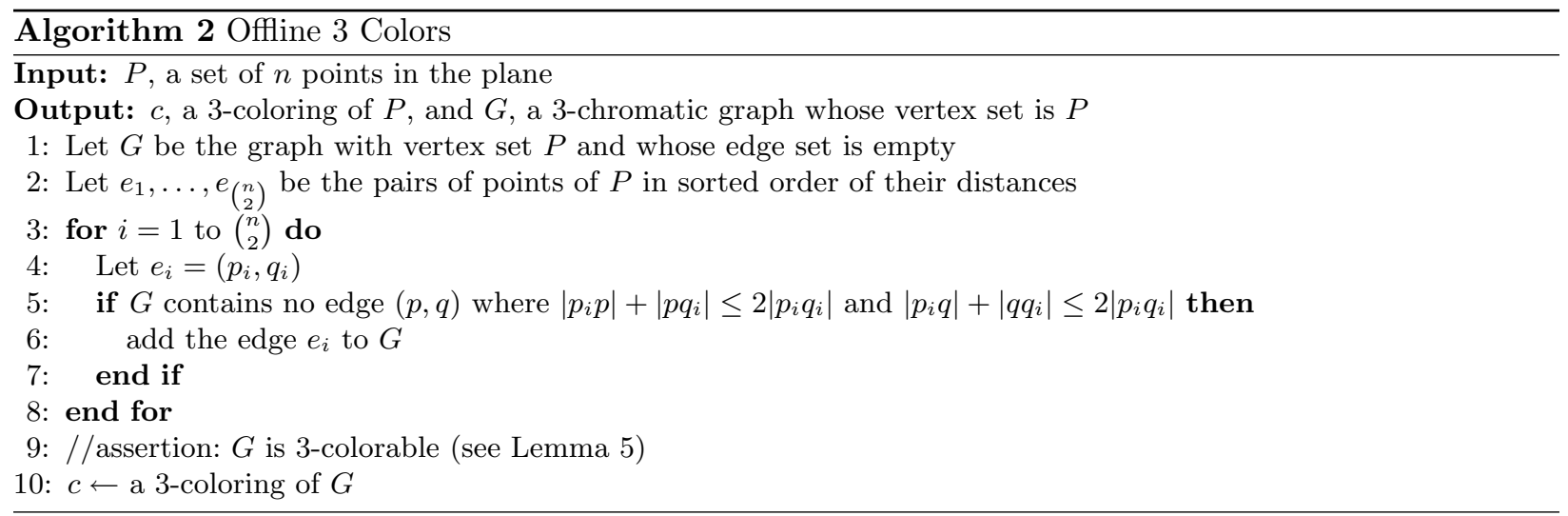


We now consider the case when $k=3$. Our strategy is to construct a graph such that any coloring of that graph has the 2-ellipse property. We then show that this graph is 3-colorable.

Lemma 3. The graph $G$ computed by Algorithm 2 is triangle-free.

Proof. Assume that $G$ contains a triangle with vertices $p, q$, and $r$. We may assume without loss of generality that $(p, r)$ was the last edge of this triangle that was considered by the algorithm. This means that $(p, r)$ is the longest edge of the triangle. When $e_{i}=(p, r)=\left(p_{i}, q_{i}\right)$ in line $4, G$ already contains the edge $(p, q)$. Since $\left|p_{i} p\right|+\left|p q_{i}\right|=|p p|+|p r| \leq 2|p r|$ and $\left|p_{i} q\right|+\left|q q_{i}\right|=|p q|+|q r| \leq 2|p r|$, the edge $(p, r)$ is not added to $G$. This is a contradiction and, therefore, $G$ is triangle-free.

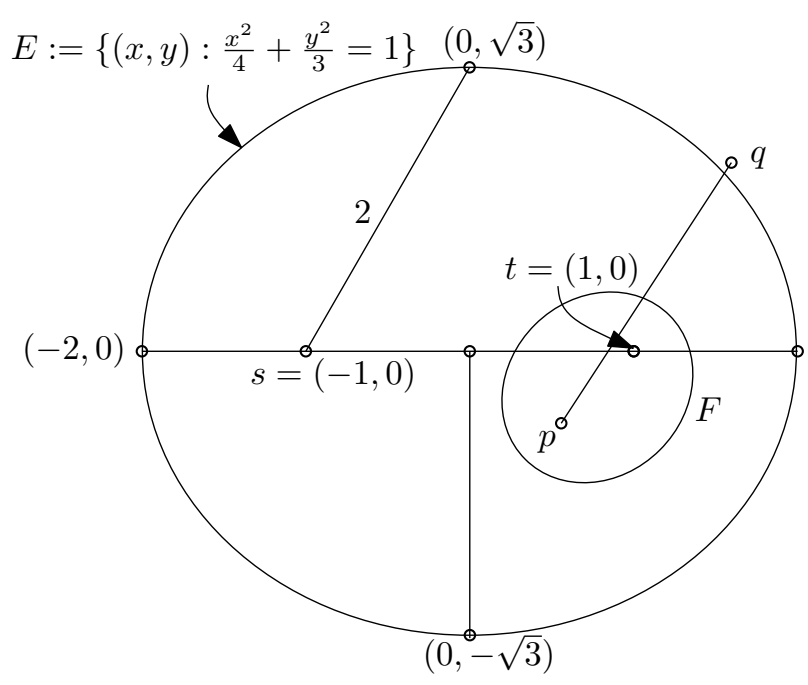

Fig. 3. Proof of Lemma 4.

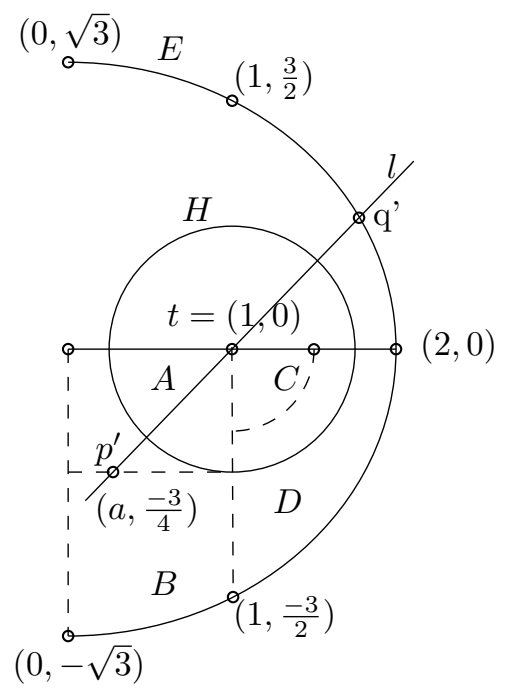

Lemma 4. The graph $G$ computed by Algorithm 2 is plane.

Proof. Refer to Figure 3 for what follows. Assume that $G$ contains two crossing edges $(p, q)$ and $(s, t)$. We may assume without loss of generality that $s=(-1,0), t=(1,0)$, and the pair $(s, t)$ has a larger index than $(p, q)$ after the pairs have been sorted in line 2. Thus, we have $|p q| \leq|s t|$. When $e_{i}=(s, t)=\left(p_{i}, q_{i}\right)$ in line 4 , $G$ already contains the edge $(p, q)$. Let $E$ be the ellipse whose boundary is determined by the set of points $e$ where $|s e|+|e t|=2|s t|$ (see Figure 3, left). If both $p$ and $q$ are outside $E$, then $|p q|>|s t|$. If both $p$ and $q$ are inside $E$, then it follows from step 2 of the algorithm that the edge $(s, t)$ is not added to $G$. Therefore, exactly one point of $\{p, q\}$ is inside $E$.

Without loss of generality, $p$ is inside $E, q$ is outside $E$, the pair $(p, t)$ has a smaller index than the pair $(p, s)$ after the pairs have been sorted in line 2 , and $p$ is below the $x$-axis. We will show below that the ellipse $F$ whose boundary is the set of points $f$ such that $|p f|+|f t|=2|p t|$ is completely contained inside $E$. Thus, since $E$ does not contain any edge when $(s, t)$ is inserted, $F$ does not contain any edge at the same time either. It follows that the edge $(p, t)$ is added to $G$, thus preventing the insertion of the edge $(s, t)$ because we claim that the edge $(p, t)$ has smaller index than $(s, t)$. To prove this claim, notice that since the ellipse $F$ is contained in the ellipse $E$ and $s \neq p$ it follows that $F$ is strictly contained in $E$. Therefore, $(p, t)$ has smaller index than $(s, t)$.

It remains to prove that the ellipse $F$ is contained in the ellipse $E$. The proof considers four cases, depending on the location of $p$ (see Figure 3, right). 
Case A: $\left[0 \leq p_{x} \leq 1\right.$ and $\left.-3 / 4 \leq p_{y} \leq 0\right]$ We show that for any point $e$ on $E$, we have $|p e|+|e t|>2|p t|$. Note that we only need to check the case when $e$ is below the $x$-axis and either $p_{y}=-3 / 4$ or $p_{x}=0$ (observe that for any $e=\left(e_{x}, e_{y}\right)$ above the $x$-axis there exists a point $e^{\prime}=\left(e_{x},-e_{y}\right)$ such that $\left.|p e|+|e t| \geq\left|p e^{\prime}\right|+\left|e^{\prime} t\right|\right)$. We consider these two cases separately.

If $p_{y}=-3 / 4$, let $a=p_{x}$. In this case, we have

$$
|p e|+|e t|-2|p t|=\sqrt{\left(a-e_{x}\right)^{2}+\left(3 / 4+e_{y}\right)^{2}}+\sqrt{\left(e_{x}-1\right)^{2}+e_{y}^{2}}-2 \sqrt{(a-1)^{2}+9 / 16} .
$$

Since $e_{y}=-\sqrt{\left(12-3 e_{x}^{2}\right)} / 2$, the above expression is completely determined by $a$ and $e_{x}$. Elementary algebraic transformations (verified with Maple) show that it always evaluates to a positive value when $e_{x}$ varies from -2 to 2 and $a$ varies from 0 to 1 .

If $p_{x}=0$, let $b=p_{y}$. We have

$$
|p e|+|e t|-2|p t|=\sqrt{e_{x}^{2}+\left(b-e_{y}\right)^{2}}+\sqrt{\left(e_{x}-1\right)^{2}+e_{y}^{2}}-2 \sqrt{1+b^{2}} .
$$

As in the previous case, when $e_{x}$ varies from -2 to 2 and $b$ varies from 0 to $-3 / 4$, elementary algebraic manipulations (which we verified with Maple) show that the above expression evaluates to a positive number.

Case B: $\left[0 \leq p_{x} \leq 1\right.$ and $\left.p_{y}<-3 / 4\right]$ In this case, we show that $|p q|>|s t|$. Let $a$ be the $x$-coordinate of $p$, let $p^{\prime}$ be the point $(a,-3 / 4)$, and let $q^{\prime}$ be the intersection of the line $l$ through $t$ and $p^{\prime}$ with the ellipse $E$. Since $|p q| \geq\left|p^{\prime} q^{\prime}\right|$, it is sufficient to show that $\left|p^{\prime} q^{\prime}\right|>|s t|=2$. The line $l$ is given by the equation

$$
y=\frac{3(x-1)}{4(1-a)} .
$$

Since $E$ is given by the equation $3 x^{2}+4 y^{2}=12$, the intersection between $E$ and $l$ is given by:

$$
4(1-a)^{2} x^{2}+3(x-1)^{2}-16(1-a)^{2}=0 .
$$

For a fixed value of $a$, let $x(a)$ be the largest root of the above polynomial, and let $y(a)$ be the $y$-coordinate of $l$ at $x=x(a)$. Then

$$
\left|p^{\prime} q^{\prime}\right|=\sqrt{(x(a)-a)^{2}+(y(a)+3 / 4)^{2}} .
$$

When $a$ varies from 0 to 1 , this expression always evaluates to strictly more than 2 .

Case C: $\left[p_{x}>1\right.$ and $\left.|p t| \leq 1 / 2\right]$ In this case, the ellipse $F$ is completely contained in the circle $H$ centered at $t$ whose radius is $3 / 2$. Since $H$ is contained in $E, F$ is also contained in $E$.

Case D: $\left[p_{x}>1\right.$ and $\left.|p t|>1 / 2\right]$ We separate this case into two subcases, depending on whether $q$ has a positive or negative $x$-coordinate. If it is positive, then the part of $\overline{p q}$ that is above the $x$-axis has length at least $3 / 2$ and the part of $\overline{p q}$ that is below the $x$-axis has length more than $1 / 2$, which means that $|p q|>2=|s t|$. If the $x$-coordinate of $q$ is negative but greater than -1 , then the same reasoning applies. If the $x$-coordinate of $q$ is smaller than -1 , then the projection of $\overline{p q}$ on the $x$-axis is larger than 2 , which means that $|p q|>2=|s t|$.

Lemma 5. The graph G computed by Algorithm 2 is 3-colorable.

Proof. By Lemmas 3 and Lemma 4, G is plane and triangle-free. It is known that such a graph is 3-colorable; see $[3],[7]$.

Lemma 6. For any point set $P$, the 3-coloring of $P$ computed by Algorithm 2 has stretch factor at most 2. Thus, we have $t(3) \leq 2$. 


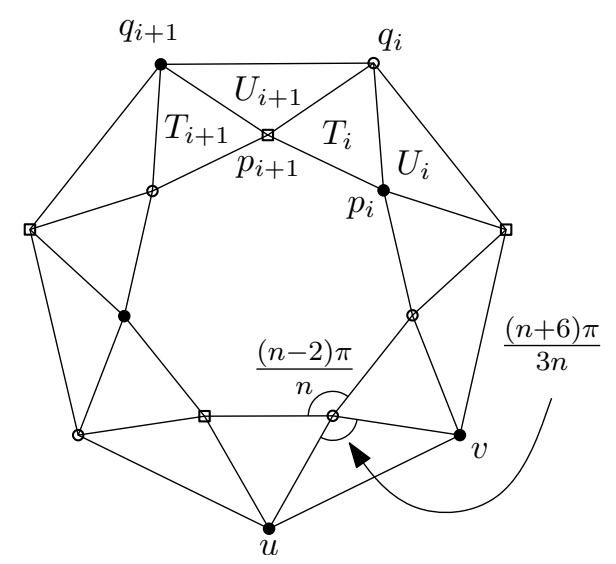

Fig. 4. Lower bound of $2-\epsilon$ for $k=3$.

Proof. It is sufficient to show that the 3-coloring $c$ produced by Algorithm 2 has the 2-ellipse property. Let $p$ and $q$ be points in $P$ such that $c(p)=c(q)$. Let $E$ be the ellipse whose boundary is the set of points $e$ such that $|p e|+|e q|=2|p q|$. Since $(p, q)$ is not an edge in $G, G$ must contain an edge $(s, t)$ whose two endpoints are inside $E$. Since $c(s) \neq c(t)$, at least one of $s$ and $t$ has a different color than $p$ and $q$. Without loss of generality, $s$ is that point. Since $s$ is inside $E$, we have that $|p s|+|s q| \leq 2|p q|$.

Lemma 7. For every $\epsilon>0$, there exists a point set $P$ such that every 3 -coloring of $P$ has stretch factor at least $2-\epsilon$. Thus, we have $t(3) \geq 2$.

Proof. Refer to Figure 4 for what follows. Let $n \equiv 2 \bmod 3$ be an integer, and let $P=\left\{p_{1}, \ldots, p_{n}, q_{1}, \ldots, q_{n}\right\}$ where the $p_{i}$ 's are the vertices of a regular $n$-gon given in counter-clockwise order, and the $q_{i}$ 's are such that the triangles $T_{i}=\left(q_{i}, p_{i}, p_{i+1}\right)$ are equilateral with interior lying outside the $n$-gon (indices are taken modulo $n$ ); Now consider the set of triangles $\mathcal{T}=\left\{T_{1}, \ldots, T_{n}, U_{1}, \ldots, U_{n}\right\}$, where $U_{i}=\left(q_{i-1}, p_{i}, q_{i}\right)$. A simple parity argument (where $n \equiv 2 \bmod 3$ ) shows that, for any 3 -coloring of $P$, there is at least one triangle of $\mathcal{T}$ that has two vertices $u$ and $v$ that are assigned the same color. If this triangle is $T_{i}$, then the stretch factor between $u$ and $v$ is at least 2. If this triangle is a $U_{i}$, then the stretch factor between $u$ and $v$ is at least $1 / \sin ((n+6) \pi / 6 n)$, which tends to 2 as $n$ goes to infinity.

Next, we consider the case when $k=4$. For this case, we simply use the Delaunay triangulation to find a 4 -coloring. We then show that this coloring satisfies the $\sqrt{2}$-ellipse property.

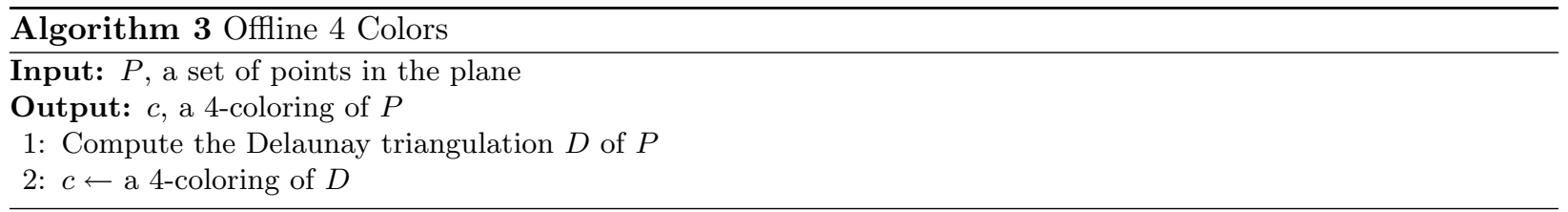

Lemma 8. For any point set $P$, the 4-coloring of $P$ computed by Algorithm 3 has stretch factor at most $\sqrt{2}$. Thus, we have $t(4) \leq \sqrt{2}$.

Proof. Refer to Figure 5 for what follows. It is sufficient to show that the coloring $c$ computed by Algorithm 3 has the $\sqrt{2}$-ellipse property. Let $p$ and $q$ be points of $P$ such that $c(p)=c(q)$. Since $(p, q)$ is not an edge in the Delaunay triangulation, the circle $C$ whose diameter is $p q$ contains at least one point of $P$. For a 


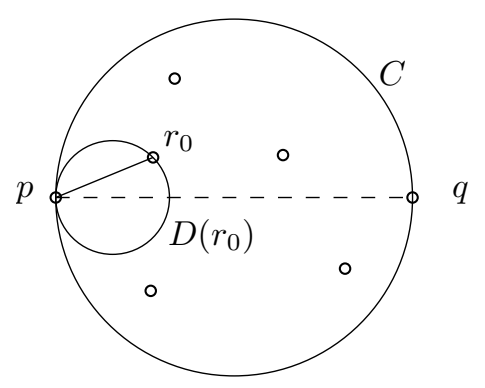

Fig. 5. Upper bound of $\sqrt{2}$ for $k=4$.

point $r$ inside $C$, let $D(r)$ be the circle through $p$ and $r$ whose center is on $p q$. Let $r_{0}$ be the point inside $C$ such that $D\left(r_{0}\right)$ has minimum diameter. Then, $D\left(r_{0}\right)$ is an empty circle with $p$ and $r_{0}$ on its boundary, which means that $\left(p, r_{0}\right)$ is a Delaunay edge. Therefore, $c\left(r_{0}\right) \neq c(p)$, and since $r_{0}$ is inside $C$, we have $\left|p r_{0}\right|+\left|r_{0} q\right| \leq \sqrt{2}|p q|$.

Lemma 9. For every $\epsilon>0$, there exists a point set $P$ such that every 4-coloring of $P$ has stretch factor at least $\sqrt{2}-\epsilon$. Thus, we have $t(4) \geq \sqrt{2}$.

Proof. Refer to Figure 6 for what follows. Let $n$ be an odd integer, and let $P=\left\{p_{1}, \ldots, p_{n}, q_{1}, \ldots, q_{n}\right\}$, where the $p_{i}$ 's are the vertices of a regular $n$-gon, the $q_{i}$ 's are the vertices of a larger regular $n$-gon with the same center, and $\left|q_{i} p_{i}\right|=\left|p_{i} p_{i+1}\right|$ for all $i$. Let $Q_{i}$ be the quadrilateral $\left(p_{i}, p_{i+1}, q_{i+1}, q_{i}\right)$. A simple parity argument shows that for any 4-coloring of $P$, there is at least one $Q_{i}$ that has two vertices $u$ and $v$ that are assigned the same color. The stretch factor between $u$ and $v$ is then at least $1 / \sin ((n+2) \pi / 4 n)$, which tends to $\sqrt{2}$ when $n$ goes to infinity.

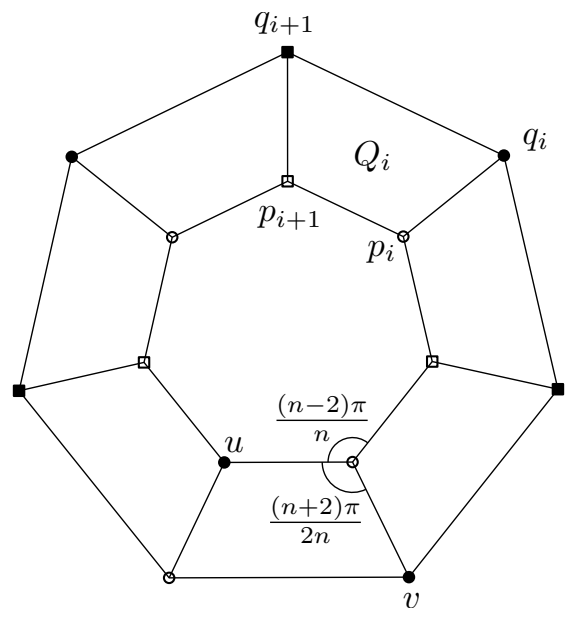

Fig. 6. Lower bound of $\sqrt{2}-\epsilon$ for $k=4$.

Our general algorithm for values $k>4$ uses ideas from the ordered $\Theta$-graph of Bose et al. [1]. We take advantage of the fact that we are in an off-line context, so that we can sort the points according to their $y$-coordinates. We process the points one by one from the lowest to the highest, splitting the half-plane below the current point $p$ being processed into $k-1$ cones of angle $\pi /(k-1)$ and having their apex at $p$. For each such cone $c_{j}$, we take the point $r_{j}$ in $c_{j}$ that is closest to $p$. Then we assign $p$ a color that has not been 
assigned to any of the $r_{j}$ 's. The fact that this algorithm uses at most $k$ colors is straightforward, since there are at most $k-1$ such $r_{j}$.

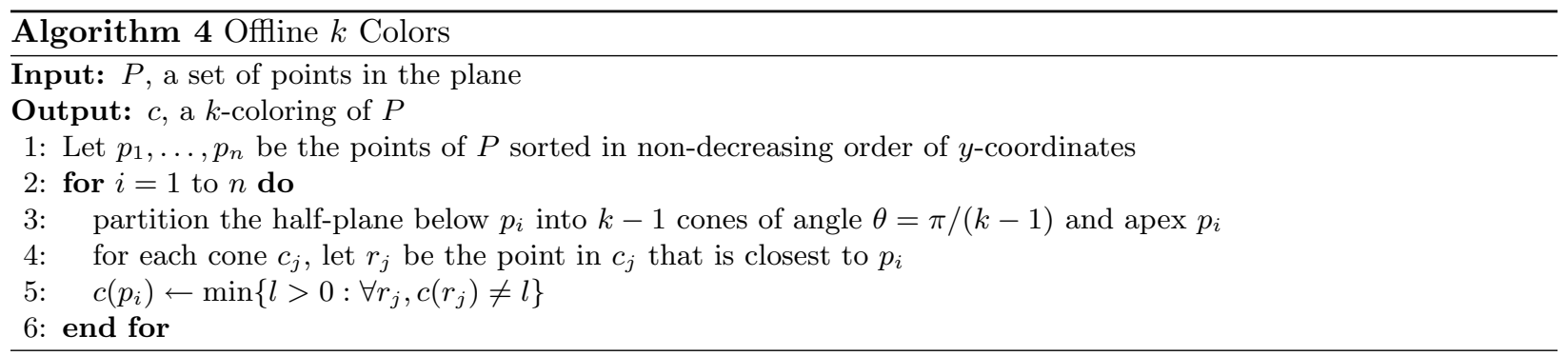

Lemma 10. For $k>4$, we have $t(k) \leq 1+2 \sin (\pi /(2 k-2))$.

Proof. Refer to Figure 7 for what follows. Let $p$ and $q$ be points of $P$ such that $c(p)=c(q)$. We may assume without loss of generality that $q_{y} \leq p_{y}$. Let $c$ be the cone with apex at $p$ that contains $q$ in line 4 of Algorithm 4, let $r$ be the nearest neighbor of $p$ in $c$, let $r^{\prime}$ be the intersection between the ray emanating from $p$ through $r$ and the circle centered at $p$ with radius $|p q|$, and let $\alpha=\angle r p q$. Then,

$$
|p r|+|r q| \leq|p r|+\left|r r^{\prime}\right|+\left|r^{\prime} q\right|=|p q|+\left|r^{\prime} q\right|=|p q|+2 \sin \frac{\alpha}{2}|p q| \leq\left(1+2 \sin \frac{\pi}{2(k-1)}\right)|p q| .
$$

It follows that the coloring computed by Algorithm 4 has the $(1+2 \sin (\pi /(2 k-2))$-ellipse property. The result follows from the fact that $c(r) \neq c(p)$ and that Algorithm 4 uses at most $k$ colors.

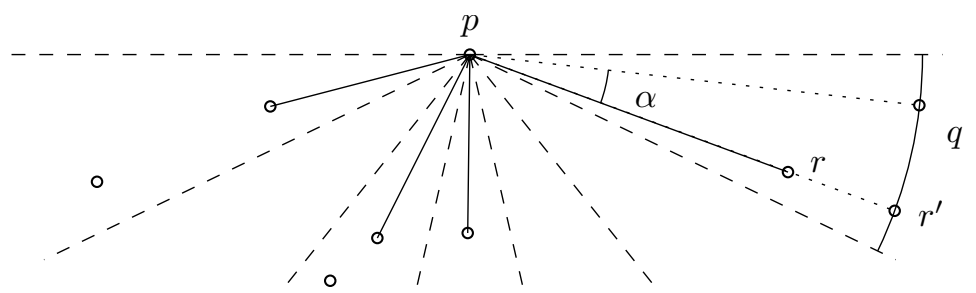

Fig. 7. Upper bound of $1+2 \sin (\pi /(2 k-2))$ for $k>4$.

Lemma 11. Let $p, q, r$ be three distinct vertices of a regular $(k+1)$-gon. Then the ratio $(|p r|+|r q|) /|p q|$ is at least $1 / \cos \left(\frac{\pi}{k+1}\right)$ and this value is achieved when $p, r$, and $q$ are consecutive vertices.

Proof. Refer to Figure 8 for what follows. For fixed $p$ and $q$, the ratio $(|p r|+|r q|) /|p q|$ is minimized when $r$ is adjacent to either $p$ or $q$. In that case, the angle $\alpha=\angle q p r=\pi /(k+1)$ (In any case where $r$ is adjacent to $q$, the angle $\alpha=\angle q p r=\pi /(k+1))$. We show that for a fixed point $p$ and three consecutive vertices $p_{i-1}, p_{i}$ and $p_{i+1}$ of the regular $(k+1)$-gon such that $\left|p p_{i-1}\right|<\left|p p_{i}\right|<\left|p p_{i+1}\right|$ the ratio $\left(\left|p p_{i-1}\right|+\left|p_{i-1} p_{i}\right|\right) /\left|p p_{i}\right|$ is smaller than $\left(\left|p p_{i}\right|+\left|p_{i} p_{i+1}\right|\right) /\left|p p_{i+1}\right|$ and the result follows.

Without loss of generality, $p_{i-1}, p_{i}$ and $p_{i+1}$ are in clockwise order. Let $p_{i-1}^{\prime}$ and $p_{i}^{\prime}$ be the rotation of $p_{i-1}$ and $p_{i}$ around $p$ by a clockwise angle of $\alpha$. Also, let $p_{i}^{*}$ be the intersection of $\overline{p p_{i}}$ with the parallel line 


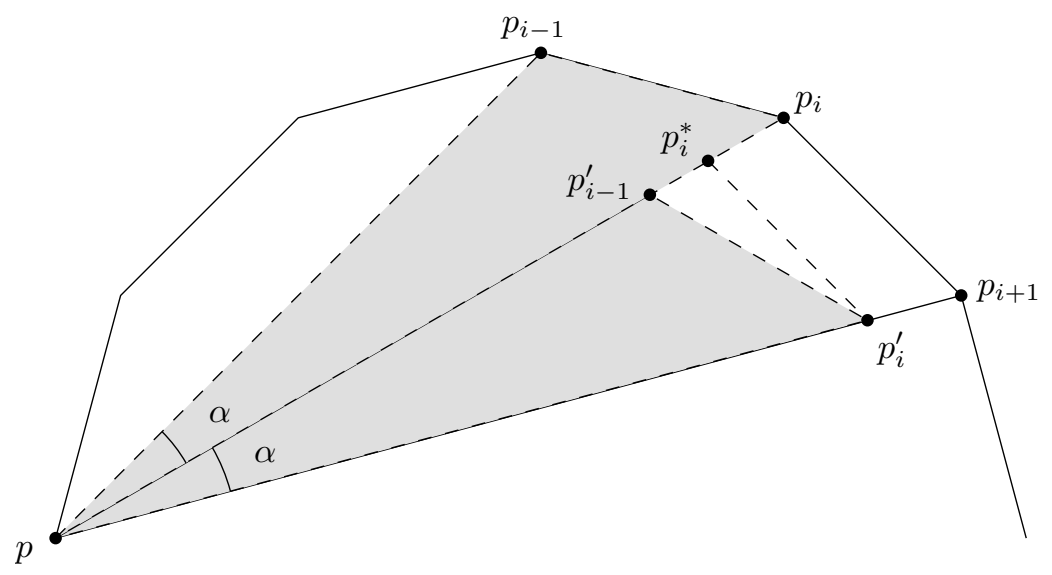

Fig. 8. Illustration of the proof of Lemma 11.

to $\overline{p_{i} p_{i+1}}$ through $p_{i}^{\prime}$. Triangle $p p_{i}^{*} p_{i}^{\prime}$ is similar to triangle $p p_{i} p_{i+1}$. Therefore,

$$
\begin{aligned}
\left(\left|p p_{i}\right|+\left|p_{i} p_{i+1}\right|\right) /\left|p p_{i+1}\right| & =\left(\left|p p_{i}^{*}\right|+\left|p_{i}^{*} p_{i}^{\prime}\right|\right) /\left|p p_{i}^{\prime}\right| \\
& =\left(\left|p p_{i-1}^{\prime}\right|+\left|p_{i-1}^{\prime} p_{i}^{*}\right|+\left|p_{i}^{*} p_{i}^{\prime}\right|\right) /\left|p p_{i}^{\prime}\right| \\
& >\left(\left|p p_{i-1}^{\prime}\right|+\left|p_{i-1}^{\prime} p_{i}^{\prime}\right|\right) /\left|p p_{i}^{\prime}\right| \\
& =\left(\left|p p_{i-1}\right|+\left|p_{i-1} p_{i}\right|\right) /\left|p p_{i}\right| .
\end{aligned}
$$

Therefore, the ratio $\left(\left|p p_{i-1}\right|+\left|p_{i-1} p_{i}\right|\right) /\left|p p_{i}\right|$ is minimized when $p_{i-1}$ is adjacent to $p$.

Lemma 12. For $k>4$, we have $t(k) \geq 1 / \cos \left(\frac{\pi}{k+1}\right)$.

Proof. Let $P=\left\{p_{1}, \ldots, p_{k+1}\right\}$ be the vertex set of a regular $(k+1)$-gon. By Lemma 11, for any three distinct points $p, q$, and $r$ in $P$, the ratio $(|p r|+|r q|) /|p q|$ is at least $1 / \cos \left(\frac{\pi}{k+1}\right)$ and this value is achieved when $p$, $r$, and $q$ are consecutive vertices.

By the pigeonhole principle, any $k$-coloring of $P$ has to assign the same color to at least two points, say $p$ and $q$. By the argument above, the stretch factor between $p$ and $q$ is at least $1 / \cos \left(\frac{\pi}{k+1}\right)$.

The constructions we have shown in this section use a quadratic number of edges since we consider the complete $k$-partite graph induced by the coloring of the points. To reduce this to a linear number of edges we apply Proposition 3, which slightly increases the stretch factor, giving us the following:

Theorem 2. The following are true:

1. For any point set $P$ in the plane, the complete $k$-partite graph induced by the $k$-coloring of $P$ computed by the above algorithms has a stretch factor at most $3,2, \sqrt{2}$, and $1+2 \sin \frac{\pi}{2(k+1)}$ for $k=2, k=3$, $k=4, k>4$, respectively.

2. For any $\epsilon>0$, there exist point sets such that no coloring algorithm can compute a $k$-coloring that has the $t$-ellipse property for $t$ smaller than $3-\epsilon, 2-\epsilon, \sqrt{2}-\epsilon$, and $1 / \cos \frac{\pi}{k+1}$ for $k=2, k=3, k=4$, $k>4$, respectively.

3. Thus, we have $t(2)=3, t(3)=2, t(4)=\sqrt{2}$, and $1+2 \sin \frac{\pi}{2(k+1)} \geq t(k) \geq 1 / \cos \frac{\pi}{k+1}$ for $k>4$.

4. It is possible to obtain a $((1+\epsilon) t(k))$-spanner that has $O(|P|)$ edges, from the coloring computed by the above algorithms.

\section{Upper and lower bounds on $t^{\prime}(k)$}

Recall that in the on-line setting, the algorithm receives the points of $P$ one at a time and assigns a color to a point as soon as it receives it. It cannot change the color of a point after this assignment. Naturally, 
this setting is more difficult which is reflected by higher bounds for $t^{\prime}(3)$ and $t^{\prime}(4)$. However, we are still able to give the exact value of $t^{\prime}(k)$ for $k=2,3,4$ and provide upper and lower bounds when $k>4$. In the online setting, we actually provide a general algorithm that is the same for all values of $k \geq 2$. Although it is similar to Algorithm 4, there are at least two important differences. First, since we are in an on-line setting, we cannot process the points in the order of their $y$-coordinates. Therefore, we have to use cones with an angle greater than $\pi /(k-1)$. If we choose the cones a priori as we do in Algorithm 4, we obtain cones whose angle is $2 \pi /(k-1)$. However, by aligning the cone's bisectors on the points that are chosen to be neighbors, we can get a slightly better stretch factor, since in this case, the angle is reduced to $2 \pi / k$.

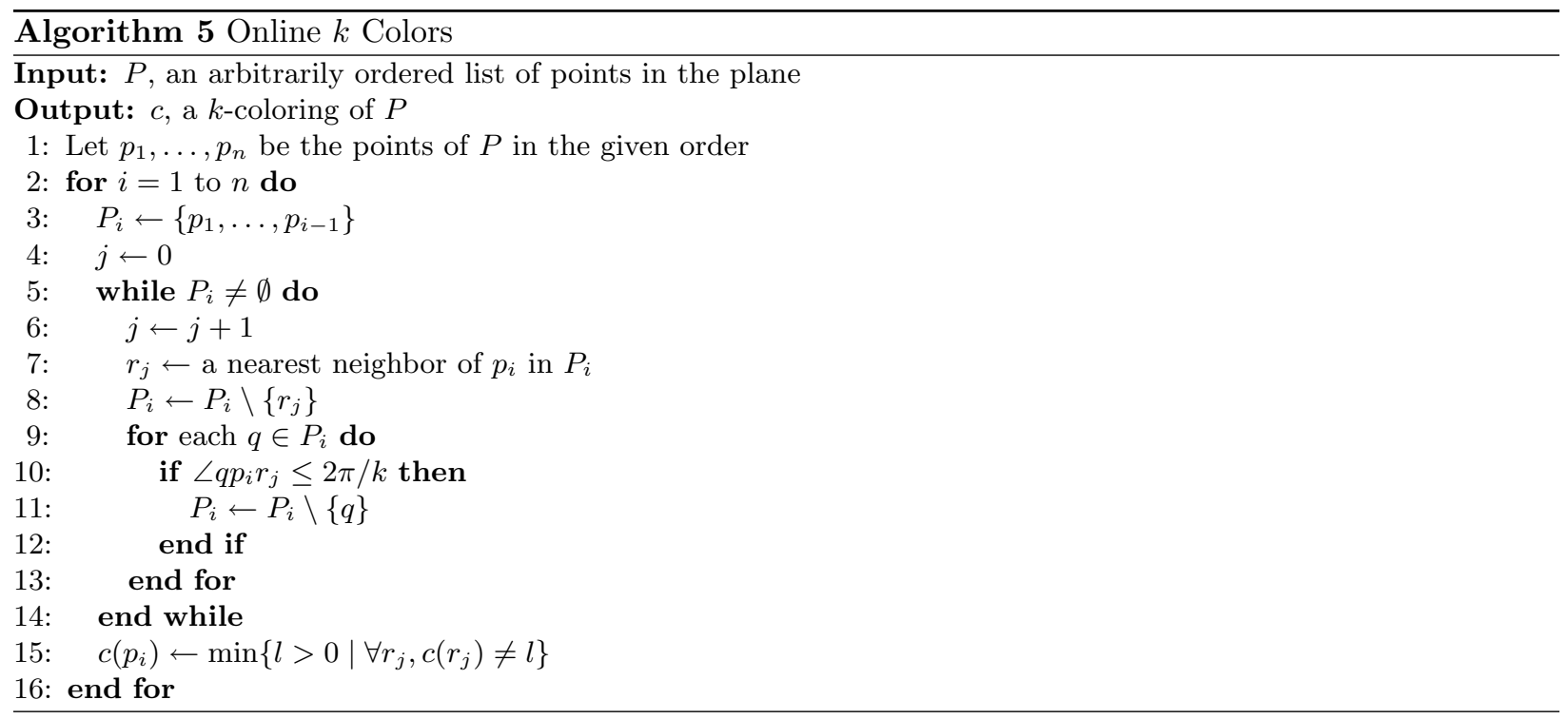

Lemma 13. For $k \geq 2$, Algorithm 5 computes a $k$-coloring that has the $t$-ellipse property for $t=1+$ $2 \sin (\pi / k)$. Thus, we have $t^{\prime}(k) \leq 1+2 \sin (\pi / k)$.

Proof. Algorithm 5 produces a $k$-coloring, because each $p_{i}$ selects at most $k-1$ points $r_{j}$. If there were more than $k-1$ such points, then two of them would form an angle of $2 \pi / k$ or less around $p_{i}$. However, this situation cannot occur because of lines 10 and 11. The proof on the stretch factor is identical to the one given in Lemma 10.

Corollary 1. We have $t^{\prime}(2) \leq 3, t^{\prime}(3) \leq 1+\sqrt{3}$ and $t^{\prime}(4) \leq 1+\sqrt{2}$.

Since an off-line lower bound also provides an on-line lower bound, we have $t^{\prime}(2) \geq t(2)=3$. It follows that $t^{\prime}(2)=3$. We now prove that Algorithm 5 is also optimal for $k=3$ and 4 .

Lemma 14. Let $\mathcal{A}$ be an arbitrary on-line coloring algorithm that guarantees a 3-coloring that has the t-ellipse property. Then its stretch factor, $t$, is at least $1+\sqrt{3}$.

Proof. Refer to Figure 9 for what follows. The proof is by an adversarial argument, where the adversary forces a stretch factor of at least $1+\sqrt{3}$. The main objective of the adversary is to force $\mathcal{A}$ to assign different colors to the vertices of an equilateral triangle. When this is achieved, the next point is placed in the center of this triangle (see Figure 9(a)). This results in a stretch factor of $1+\sqrt{3}$, and the adversary is done.

Consider Figure 9(b), where the points are numbered by the order of insertion. Up to symmetry, there is only one way to assign colors to points $p_{1}$ to $p_{6}$ such that $t<1+\sqrt{3}$ (e.g., $c\left(p_{1}\right)=$ red, $c\left(p_{2}\right)=b l u e, c\left(p_{3}\right)=$ red, $c\left(p_{4}\right)=$ green, $c\left(p_{5}\right)=$ green, $c\left(p_{6}\right)=$ green $)$. The key property is that the points $p_{4}, p_{5}$ and $p_{6}$ must 

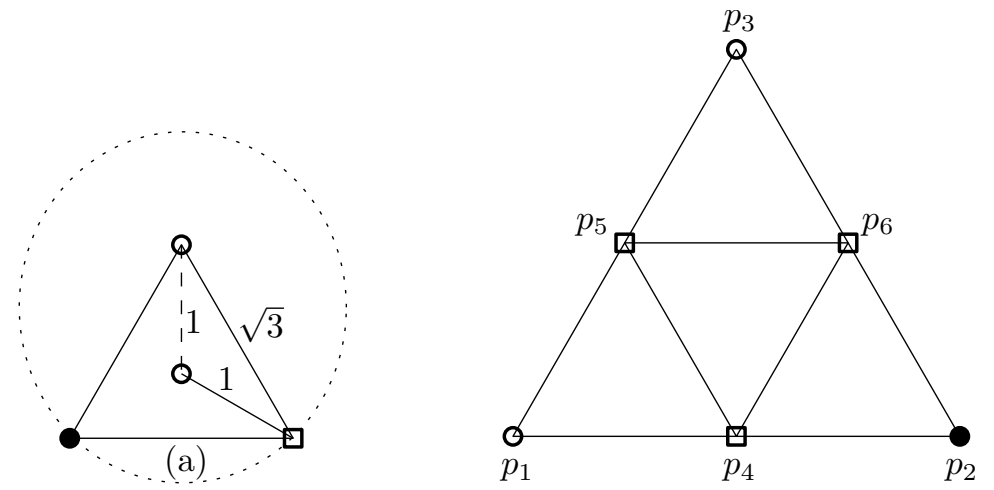

(b)

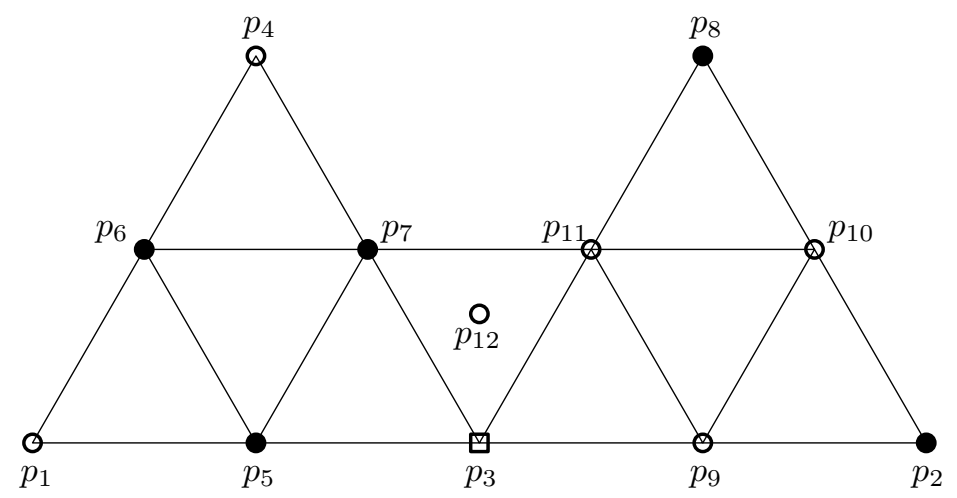

(c)

Fig. 9. Online lower bound of $1+\sqrt{3}$ for $k=3$. 
be assigned the same color that is different from the colors assigned to the first three points. If any of these conditions is violated, then the spanning ratio is at least $1+\sqrt{3}$, either by stopping at some time in the insertion order or because the corners of an equilateral triangle are assigned three different colors.

Next, consider Figure 9(c), where the point set of Figure 9(b) is reproduced twice. Consider triangles $\triangle\left(p_{3}, p_{5}, p_{7}\right), \triangle\left(p_{3}, p_{9}, p_{11}\right)$ and $\triangle\left(p_{3}, p_{7}, p_{11}\right)$ after the insertion of $p_{11}$. At least one of these triangles has to be assigned three different colors, otherwise, the stretch factor would already be $1+\sqrt{3}$. Assume w.l.o.g. that triangle $\triangle\left(p_{3}, p_{7}, p_{11}\right)$ is assigned three different colors then by the insertion of point $p_{12}$ in the center of the triangle, we force a spanning ratio of $1+\sqrt{3}$, as required.

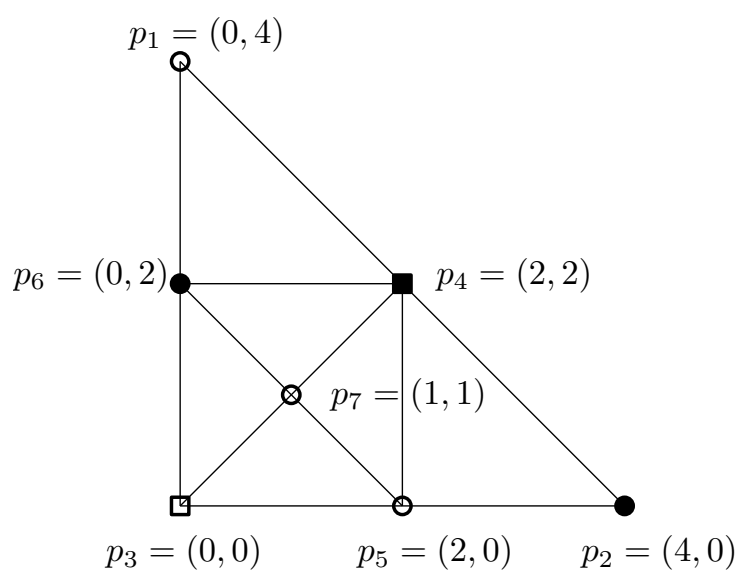

Fig. 10. Online lower bound of $1+\sqrt{2}$ for $k=4$.

Lemma 15. Let $\mathcal{A}$ be an arbitrary on-line coloring algorithm that guarantees a 4-coloring that has the $t$-ellipse property. Then the stretch factor, $t$ is at least $1+\sqrt{2}$.

Proof. Consider the point set depicted in Figure 10. $\mathcal{A}$ must assign different colors to $p_{3}, p_{4}, p_{5}$ and $p_{6}$, otherwise the stretch factor will already be greater than $1+\sqrt{2}$. Upon introduction of $p_{7}, \mathcal{A}$ must assign it the same color as one of $p_{3}, p_{4}, p_{5}$ or $p_{6}$. The stretch factor between $p_{7}$ and that point is $1+\sqrt{2}$.

Lemma 16. Let $\mathcal{A}$ be an arbitrary on-line coloring algorithm that guarantees a $k$-coloring that has the $t$-ellipse property. Then the stretch factor, $t$, is at least $1 / \cos \left(\frac{\pi}{k}\right)$.

Proof. Let $P=\left\{p_{1}, \ldots, p_{k}, q\right\}$, where the $p_{i}$ are the vertices of a regular $k$-gon $K$ and $q$ is the center of the circumcircle of $K$. If, after processing $p_{1}$ to $p_{k}, \mathcal{A}$ assigned the same color to two points, then as in Lemma 12, the stretch factor is at least $1 / \cos \left(\frac{\pi}{k}\right)$. Otherwise, all $p_{i}$ are assigned different colors. When $q$ is introduced, the color $\mathcal{A}$ assigns to it has already been assigned to some other point $p$. In that case, the stretch factor for the edge $(q, p)$ is $1+2 \sin (\pi / k)$. We claim that this is at least $1 / \cos \left(\frac{\pi}{k}\right)$ (for $k>4$ ). Indeed, for $0<x<\pi / 4$, we have

$$
\begin{aligned}
1+2 \sin x & =\frac{1}{\cos x}(\cos x+2 \sin x \cos x) \\
& >\frac{1}{\cos x}\left(\cos ^{2} x+2 \sin ^{2} x\right) \\
& >\frac{1}{\cos x}\left(\cos ^{2} x+\sin ^{2} x\right) \\
& =\frac{1}{\cos x} .
\end{aligned}
$$


The constructions we have shown in this section use a quadratic number of edges since we consider the complete $k$-partite graph induced by the coloring of the points. To reduce this to a linear number of edges we apply Proposition 3, which slightly increases the stretch factor, giving us the following:

Theorem 3. The following are true:

1. For any sequence $P$ of points in the plane, the complete $k$-partite graph induced by the on-line $k$-coloring of $P$ computed by the above algorithms has a stretch factor at most $3,1+\sqrt{3}, 1+\sqrt{2}$, and $1+2 \sin \frac{\pi}{k}$ for $k=2, k=3, k=4, k>4$, respectively.

2. For any $\epsilon>0$, there exist point sets such that no on-line coloring algorithm can compute an on-line $k$-coloring that has the $t$-ellipse property for $t$ smaller than $3-\epsilon, 1+\sqrt{3}-\epsilon, 1+\sqrt{2}-\epsilon$, and $1 / \cos \frac{\pi}{k}$ for $k=2, k=3, k=4, k>4$, respectively.

3. Thus, we have $t^{\prime}(2)=3, t^{\prime}(3)=1+\sqrt{3}, t^{\prime}(4)=1+\sqrt{2}$, and $1+2 \sin \frac{\pi}{k} \geq t^{\prime}(k) \geq 1 / \cos \frac{\pi}{k}$ for $k>4$.

4. It is possible to obtain a $\left((1+\epsilon) t^{\prime}(k)\right)$-spanner that has $O(|P|)$ edges, from the coloring computed by the above algorithms.

\section{Simulation Results}

Using simulation, we now provide estimates of the average stretch factor of the colorings produced by Algorithm 4 and Algorithm 5. Using a uniform distribution, we generated 200 sets of 50 points and 200 sets of 200 points. For each point set, we computed the stretch factor for $k$ ranging from 2 to 10 . Figure 11 and Figure 12 show the results we obtained for the stretch factor. The $95 \%$ confidence interval for these values is \pm 0.0365 .

The general behavior of the average case performance ratio of these algorithms is not much different than what can be expected from the worst case analysis. In particular, the off-line algorithm performs significantly better than the on-line algorithm. Also, in both cases, as $k$ increases, the stretch factor reduction becomes less and less important. Another interesting observation is that for $k$ large enough $(k>6$ for 50 points and $k>3$ for 200 points), the average case stretch factor of the on-line algorithm is worse than the worst case stretch factor of the off-line algorithm.

For $k=2,3$ and 4, in the off-line case, we used the algorithm for general values of $k$. It is interesting to notice that for $k=4$, the average stretch factor obtained using Algorithm 4 is greater than the worst case stretch factor obtained using Algorithm 3. However, Algorithm 3 is less practical, since we have to compute a 4-coloring of a planar graph.

\section{Conclusion}

In this paper, we investigated the problem of computing a spanner of a point set that has chromatic number $k$. To the best of our knowledge, this problem has not been considered before. For small values of $k(k \leq 4)$, we provided tight upper and lower bounds on the smallest possible stretch factor of such spanners. For larger values of $k$, we provided general upper and lower bounds which, unfortunately, are not tight. Our construction algorithms show how to color a point set with $k$ colors such that the complete $k$-partite graph induced by this coloring has the stated stretch factor. The number of edges in these graphs can be reduced from quadratic to linear with a slight increase in the spanning ratio by applying the general technique of Gudmundsson et al. [4]. An interesting open problem in this setting of the problem is to find tight upper and lower bounds when $k>4$.

We also considered an on-line variant of this problem where the points are presented sequentially and our algorithm assigns a color to each point upon reception such that the complete $k$-partite graph induced by the coloring is a constant spanner. Again, for small values of $k(k \leq 4)$, we provided tight upper and lower bounds on the smallest possible stretch factor of such spanners and for $k>4$, we provided general upper and lower bounds that are not tight. A linear-sized spanner can be constructed after all the points have been colored by applying the technique of Gudmundsson et al. [4]. However, in this case, our algorithm 


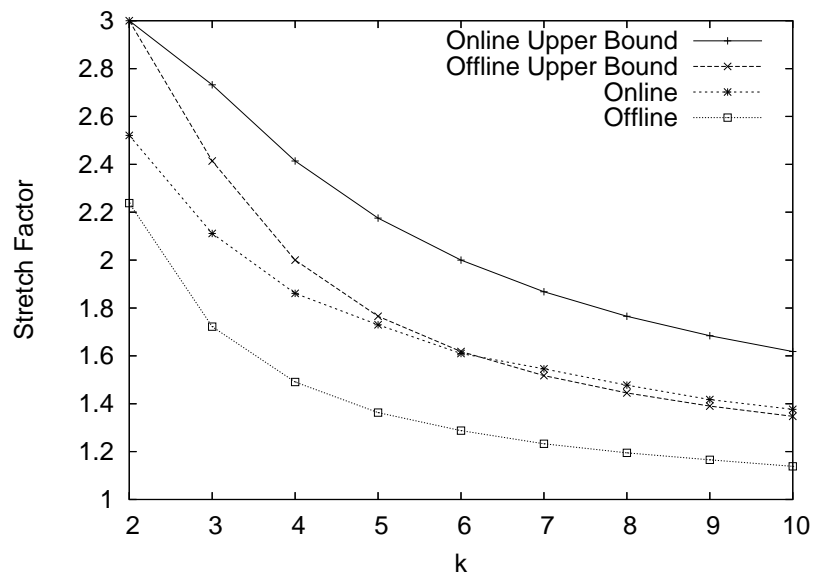

\begin{tabular}{|l|ll|l|ll|}
\hline$k$ & offline online & $k$ & offline online \\
\hline 2 & 2.2383 & 2.5208 & 7 & 1.2329 & 1.5456 \\
3 & 1.7219 & 2.1111 & 8 & 1.1947 & 1.4778 \\
4 & 1.4907 & 1.8608 & 9 & 1.1658 & 1.4175 \\
5 & 1.3631 & 1.7300 & 10 & 1.1384 & 1.3765 \\
6 & 1.2877 & 1.6098 & & & \\
\hline
\end{tabular}

Fig. 11. Simulation results for 50 nodes using Algorithm 4 and Algorithm 5.

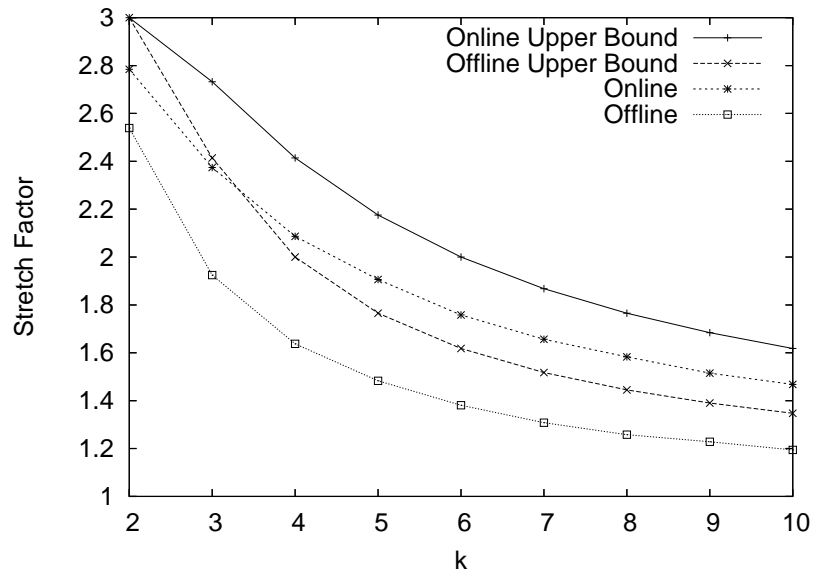

\begin{tabular}{|l|ll|l|ll|}
\hline$k$ & offline online & $k$ & offline online \\
\hline 2 & 2.5390 & 2.7844 & 7 & 1.3079 & 1.6563 \\
3 & 1.9245 & 2.3743 & 8 & 1.2579 & 1.5833 \\
4 & 1.6377 & 2.0866 & 9 & 1.2283 & 1.5149 \\
5 & 1.4831 & 1.9062 & 10 & 1.1945 & 1.4677 \\
6 & 1.3809 & 1.7579 & & & \\
\hline
\end{tabular}

Fig. 12. Simulation results for 200 nodes using Algorithm 4 and Algorithm 5 . 
for computing the linear-sized constant spanner is not on-line. Therefore, there are two open problems in the on-line setting. First, to close the gap between the upper and lower bound for $k>4$. Second, provide an on-line algorithm that computes the linear-sized constant spanner.

\section{References}

1. Prosenjit Bose, Joachim Gudmundsson, and Pat Morin. Ordered theta graphs. Comput. Geom. Theory Appl., 28(1):11-18, 2004.

2. P. B. Callahan and S. R. Kosaraju. A decomposition of multidimensional point sets with applications to $k$-nearestneighbors and $n$-body potential fields. Journal of the ACM, 42:67-90, 1995.

3. H. Grötzsch. Ein Dreifarbensatz für dreiecksfreie Netze auf der Kugel. Wiss. Z. Martin-Luther-Univ. HalleWittenberg Math.-Natur. Reihe, 8:109-120, 1959.

4. J. Gudmundsson, C. Levcopoulos, G. Narasimhan, and M. Smid. Approximate distance oracles for geometric graphs. In Proceedings of the 13th ACM-SIAM Symposium on Discrete Algorithms, pages 828-837, 2002.

5. Giri Narasimhan and Michiel Smid. Geometric Spanner Networks. Cambridge University Press, New York, NY, USA, 2007.

6. Bhaskaran Raman and Kameswari Chebrolu. Design and evaluation of a new mac protocol for long-distance 802.11 mesh networks. In MobiCom '05, pages 156-169, New York, NY, USA, 2005. ACM Press.

7. Carsten Thomassen. A short list color proof of Grotzsch's theorem. Journal of Combinatorial Theory B, 88:189192, 2003. 